\title{
Job Embeddedness to Citizenship Behavior: Role of Outcome Orientation and Relationships with Peers
}

\author{
Satvir Singh \\ Angelo State University \\ Yilu Wang \\ University of Texas at EI Paso \\ Prajya R. Vidyarthi \\ University of Texas at EI Paso
}

While highly embedded individuals possess disproportionate resources to retain stable status, little is known about their exchange relationships with peers (TMX), especially in an outcome-oriented workgroup climate that advocates results. Drawing on theories of conservation of resources and social exchange, we advance an explanation on why highly embedded employees are likely to form high quality TMX, and how outcome-oriented workgroup climate moderates this effect. Results from a multilevel study of 297 employees nested in 49 workgroups indicated that focal employees' job embeddedness predicted their helping behaviors through their TMX, and these effects were stronger in a high outcome-oriented workgroup climate.

Keywords: job embeddedness, team-member exchange, outcome orientation, conservation of resources, social exchange

\section{INTRODUCTION}

Job embeddedness offers unique perspective to explain why people stay with their current organization, and represents broad forces on employee retention, which have been described as "net" or "web" effects (Mitchell, Holtom, Lee, Sablynski, \& Erez, 2001). The concept of job embeddedness implies that employees who are more embedded in an organization are rooted in a more complex social "web" than other less embedded individuals, and thus are able to access more information and resources, and have more opportunities (Lincoln \& Miller, 1979; Zhang \& Chen, 2010). While organizations pay enormous efforts to retain employees, especially those considered to be best fit to the current job, the disproportionate resources allocation and asymmetry of membership status between more embedded and less embedded individuals may facilitate unique social consequences. As work grows more relational and requires frequent workgroup interactions (Campbell, Liao, Chuang, Zhou, \& Dong, 2017; Grant \& Parker, 2009; Griffin, Neal, \& Parker, 2007), it is valuable to expand the current understandings of job 
embeddedness as an "antiwithdrawal" construct, and investigate social experiences and behaviors of highly embedded individuals in a workgroup setting.

The interactions between highly embedded individuals and peers, however, have been neglected in the job embeddedness literature. As work grows more relational and requires frequent workgroup exchanges (Campbell et al., 2017; Grant \& Parker, 2009; Griffin et al., 2007), it is important to expand the current understanding of job embeddedness, and investigate social experiences and consequences of highly embedded individuals and rest of the members in a workgroup setting. More importantly, studying the team-member exchange relationships of highly embedded individuals can contribute to the theoretical enrichment of the job embeddedness and organizational citizenship behaviors (OCBs) literatures (Holtom \& Sekiguchi, 2018). Holtom and Sekiguchi (2018), in addition to reaffirming that the job embeddednessOCBs relationship yields one of the most interesting and promising areas of research, called for empirical research to measure certain motives for specific type of OCBs as mediator to enrich and to further theorize the effectiveness of job embeddedness. Scholars have explored and laid important foundations for the job embeddedness-OCBs linkage (e.g., Lee, Mitchell, Sablynski, Burton, \& Holtom, 2004). Nevertheless, their explanations of OCBs' motives were constrained in the concept of job embeddedness per se, and assume that OCBs are naturally exerted by embedded individuals within a consistent "web" that they get stuck in. This overlooked the possibility that members, including those outside the "web," would choose to develop high quality relationships with highly embedded employees and become the beneficiary. Also, it would be difficult to identify and theorize why certain forms of citizenship behaviors (e.g., behaviors that benefit individuals, or the organization in general) are linked to job embeddedness without sufficiently considering the underlying mechanism and indirect effect of job embeddedness on OCBs (Holtom \& Sekiguchi, 2018; Podsakoff, MacKenzie, Paine, \& Bachrach, 2000). Furthermore, Holtom and Sekiguchi (2018) suggested that job embeddedness scholars should explore and assess a broad spectrum of situational factors that strengthen or weaken the relationship between job embeddedness and OCBs. To our knowledge, the understanding of contextual factors that may alter the job embeddedness and OCBs relationship is surprisingly underdeveloped. There is a need to further explore outcomes of job embeddedness in "less people centered" contexts, such as the outcome-oriented climate (O'Reilly, Chatman, \& Caldwell, 1991).

In an effort to respond to the call for enriching the current knowledge on the job embeddedness-OCBs relationship, we emphasize on one form of OCBs - helping behavior directed toward individuals (OCBI), and extend our above discussed research interests on team-member exchange relationship of highly embedded individuals by proposing the mediation model that job embeddedness will positively affect OCBI through team-member exchange (TMX). In this proposed model, TMX reflects the overall quality of the reciprocal relationship between a member and fellow team members (Seers, 1989), and OCBI refers to an individual's interpersonal helping behaviors that can directly benefit his or her coworkers (Williams \& Anderson, 1991). We grounded our arguments on social exchange theory (Blau, 1964) and conservation of resources theory (Hobfoll, 1989). Social exchange theory describes the rule of reciprocal exchange between two parties (Blau, 1964; Cropanzano \& Mitchell, 2005; Gouldner, 1960), and conservation of resources theory indicates that people strive to protect and develop their resources (Hobfoll, 1989, 2001). By integrating both theories, we contend that bidirectional social exchange that forms TMX is likely to be developed between highly embedded employees and peers, because peers are less likely to "beat" highly embedded individuals, and thus are likely to "join" highly embedded employees for the benefit of resources; driven by the norm of reciprocity, embedded employees who are recipients of peers' support are obligated to reciprocate helping the exchange process; such phenomena would be sustained and strengthened in an outcome-oriented workgroup climate that urges resources for result achievement.

We contribute to existing literature in several ways. First, we join and extend research on job embeddedness and OCBs by introducing TMX as the linkage and expand understanding of the formation of the reciprocal relationships between embedded employees and peers. Second, we advance theory on TMX by identifying job embeddedness as an antecedent to TMX. Antecedents to TMX are largely absent, with the exceptions of few studies. For instance, Banks et al. (2014) noted the positive correlation 
between leader-member exchange and TMX; similarly, Tse, Dasborough, and Ashkanasy (2008) suggested that high-quality leader-member exchange relationship may have implications for high-quality TMX by facilitating workplace friendship. Our study reveals the underlying mechanism that explains TMX using conservation of resources theory and social exchange theory. Finally, our study provides empirical evidence that outcome orientation as a team climate can facilitate cooperation between highly embedded individuals and rest of the coworkers. Outcome orientation is reported as one of the most prominent organizational characteristics in the business world (e.g., Baird, Jia Hu, \& Reeve, 2011), but our understandings of its effects are mostly theoretical. Conceptually, outcome orientation has been defined as an achievement centered culture or climate, and it has been coupled with terms such as "competitiveness" and "aggressiveness" (Baird et al., 2011; O'Reilly et al., 1991). However, some scholars argue that emphasizing on achievement does not necessarily make outcome orientation a negative dimension, because employees may feel that their relationships with their organization are more transactional and they will seek social exchange opportunities with their supervisor or peers (Cole, Schaninger Jr, \& Harris, 2002; Robbins, Ford, \& Tetrick, 2012). Our results show that outcome orientation can actually strengthen highly embedded individuals' social exchange relationships with their peers, which offered empirical support to this perspective.

\section{THEORY AND HYPOTHESES}

\section{An Integration of Social Exchange and Conservation of Resources Theories}

Social exchange theory emphasizes on "giving" and "returning" from parties involved in the social exchange (Blau, 1964). Social exchange relationships result in social behaviors (Homans, 1958). Under the norm of reciprocity, the process of transactions has to be bidirectional and mutually contingent, and it develops obligations, such that certain actions initiated by one party for the other party's interests generate the obligation for the other party to repay the favor that helps reach the expectancy of a fair equilibrium in the social exchange relationships (Cropanzano \& Mitchell, 2005; Gouldner, 1960). In the management literature, social exchange has been majorly conceptualized to explain the employeeorganization relationships. For instance, when employees perceive favorable support from the organization, this perception creates employees' obligation to reciprocate the organization by higher inrole performance, extra role behaviors and lower turnovers (e.g., Anand, Vidyarthi, Liden, \& Rousseau, 2010; Eisenberger, Huntington, Hutchison, \& Sowa, 1986; Eisenberger, Stinglhamber, Vandenberghe, Sucharski, \& Rhoades, 2002).

Prior literature (Liden, Wayne, \& Sparrowe, 2000; Seers, 1989) has well documented that teammember exchange (TMX) reflects the quality of social exchange relationships within a workgroup. Though not a dyadic construct, TMX as a continuum measure depicts a focal employee's perception of his or her social exchange relationship with peers: low-quality TMX implies limited resource exchange, whereas high-quality TMX is characterized by mutual trust and respect, cooperation, collaboration and social rewards, and social exchanges that extend beyond the conditions necessary for task completion (Anand et al., 2010; Farmer, Van Dyne, \& Kamdar, 2015; Liden et al., 2000; Scott \& Bruce, 1994; Seers, 1989). Empirical work has acknowledged TMX as an antecedent to positive individual outcomes such as job satisfaction (Seers, 1989), commitment and job performance (Liden et al., 2000), and citizenship behavior (Chiaburu \& Harrison, 2008; Love \& Forret, 2008).

While social exchange theory can be used to explain TMX and associated outcomes, the underlying mechanism that forms TMX has not been explicitly discussed in social exchange literature (Tse et al., 2008). For instance, Banks et al. (2014) suggested that, as employees who value high-quality social exchange relationships are likely to input their efforts to that end with respect to both their leaders and coworkers, employees who establish high-quality relationships with their leaders will also establish highquality TMX. Similarly, Tse et al. (2008) argued that high-quality leader-member exchange relationships may have implications for high-quality TMX by facilitating workplace friendships, because employees who enjoy mutual trust and respect, commitment and influence rooted in high-quality relationships with leaders will be motivated to extend or transform such high-quality relationships into friendships with 
others. However, these studies have only implicitly described the phenomena that can lead to high-quality TMX, but not sufficiently explained how the reciprocal exchange relationship is formed among team members.

The current study employs conservation of resources theory to uncover the mechanism of TMX's formation, and integrates social exchange theory and conservation of resources theory to explain the job embeddedness-OCBI relationship (Lee et al., 2004) by introducing TMX as a mediator, and to investigate these relationships in the outcome orientation workgroup climate (see Figure 1). Conservation of resources theory depicts that people actively identify challenges and advantages in their social environment, and strive to retain and develop their personal, social, and material resources (Hobfoll, 1989, 2001). In their study of social consequences for high performers, Campbell et al. (2017) drew from conservation of resources theory and found that high performers will attract social support from their coworkers if they are perceived by their peers as benefit to the resources. Because conservation of resources can be utilized to tangibly specify why a focal employee, in our case the highly embedded employee, draws reactions from peers (Campbell et al., 2017), the integration of social exchange theory and conservation of resources theory enables more comprehensive understandings of the formation of bidirectional transaction rooted in TMX. Figure 1 presents the theoretical model of this study.

\section{FIGURE 1 \\ THEORETICAL MODEL}

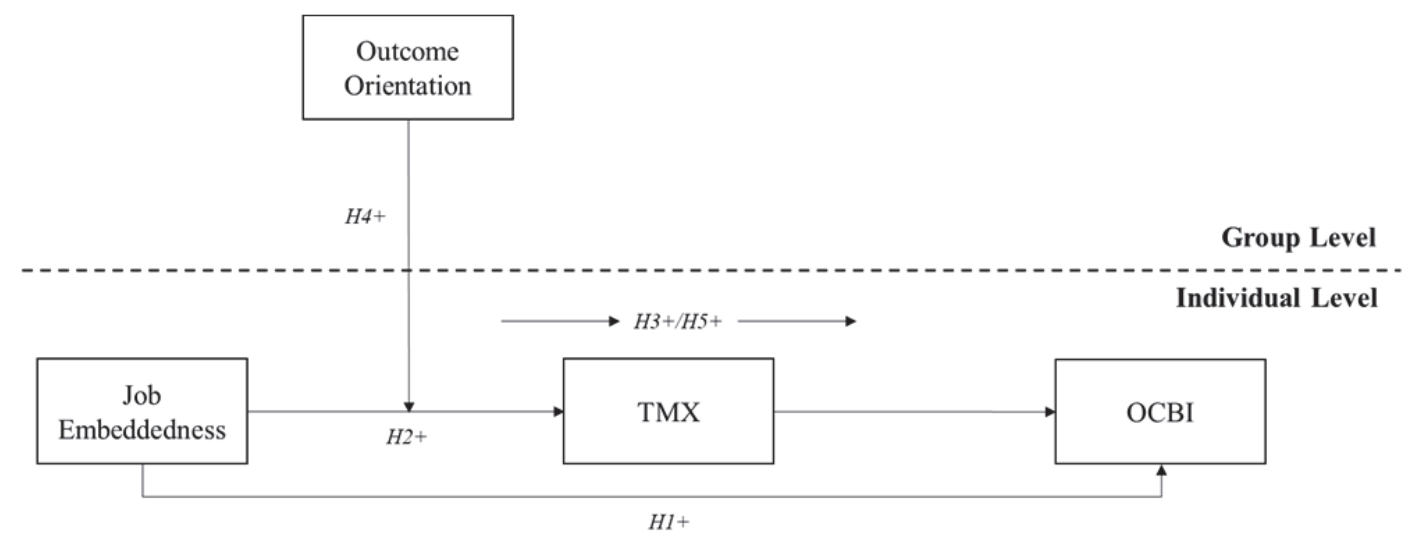

\section{Job Embeddedness, TMX, and OCBI}

Job embeddedness was theorized as a construct comprising contextual and perceptual forces that bind individuals to their current jobs and workplaces (Crossley, Bennett, Jex, \& Burnfield, 2007; Yao, Lee, Mitchell, Burton, \& Sablynski, 2004). Reflecting the idea of an individual being stuck in "a social web," Mitchell and colleagues (2001) developed three key dimensions and labeled these dimensions "links," "fit," and "sacrifice": (1) the extent to which individuals have "links" to other people or activities, (2) the extent to which people's jobs and communities are similar to or "fit" with the other aspects in their life spaces, and, (3) the ease with which links can be broken - what people would "sacrifice" if they left, especially if they had to leave their current settings. Also focusing on explaining why employees stay, Crossley et al. (2007) developed a global measure of job embeddedness. Different from Mitchell et al.'s (2001) composite measure that examines "links," "fit," and "sacrifice," Crossley et al.'s (2007) global measure integrates the recognized factors that form the overall perception of an embedded individual, and emphasizes on the end product of the mental process during which the respondent would subjectively evaluate various facets without omitting relevant information that has not been captured by facet-level scales. The empirical evidence of Crossley et al.'s (2007) study suggested that both composite and global measures of embeddedness provided meaningful predictions in retention.

In this study we attempt to establish job embeddedness as the antecedent to TMX. Considering that Mitchell et al.'s (2001) composite measure directly assesses an employee's "links" in the organization 
(e.g., How many coworkers do you interact with regularly?), we adopted Crossley et al.'s (2007) reflective measure to avoid the potential conceptual overlaps between Mitchell et al.'s (2001) job embeddedness and TMX. Even though job embeddedness was developed as an "antiwithdrawal" construct, and captures the degree of ease that an individual perceives to leave the current workplace, we argue that this degree of ease also reflects how much value does staying in the existing setting mean to the individual, and in other words, the amount of resources - for example, connections in the organization, values and recognitions gained from current jobs - that the individual could possess by retaining in the current workplace.

As mentioned above, the tenet that Hobfoll's conservation of resources theory is based on is that individuals are activated to protect their current resources and acquire new resources, and resources can be referred to as objects, states, conditions, and other things that people value (Halbesleben, Neveu, Paustian-Underdahl, \& Westman, 2014; Hobfoll, 1989, 2001). Conservation of resources theory has been used to explain a variety of workplace behaviors. For instance, in terms of the application of the resources acquisition aspect, Halbesleben and Bowler (2007) revealed an intriguing pattern that individuals who performed poorly in-role are likely to input more effort in extra-role behaviors targeting at colleagues and supervisors as an investment to exchange for resources to stop further resources losing. Utilizing both the conservation and acquisition aspects of conservation of resources theory, Campbell et al. (2017) noted that peers are likely to recognize the potential of higher performers in the workplace to enlarge the size of their resources pool, but also to drain their own resources by possessing disproportionately large proportion of the resource pool, and thus peers could support or undermine high performers resulting from different perceptions.

In this study, conservation of resources theory, together with social exchange theory, offers important insight to explain the bidirectional transactions of TMX. We argue that, highly embedded employees would view themselves as owning favorable amount of resources, and be viewed by their coworkers as occupants of resources. According to the concept of job embeddedness, the quality of connections in the organization forms employees' perceived ease to quit. Thus, it is reasonable to expect that highly embedded employees would own more high-quality social connections in the organization, and these connections would generate a larger portion of resources in the workplace pool. Similar to but different from high performers discussed by Campbell et al. (2017), the group of which does not necessarily "fit" to the workplace and owns the solid social connections as highly embedded employees do, highly embedded employees have more stable status in the organization due to their fit and possessed connections; even though it is possible for them to be recognized by peers as the potential threat to their resources, it would be much more difficult for relatively low embedded peers to substantively undermine highly embedded employees' positions. Subsequently, we expect that, since "conservation" - the option of resources protection - would mean less to peers, they would exert "accumulation" and initiate exchange targeting at highly embedded employees' resources by inputting certain investment such as helping and extra-role behavior, which forms one direction of the reciprocal relationship; ruled by social exchange theory, highly embedded employees would be obligated to repay peers' investment, and would complete the cycle of reciprocity in the coming future.

On the other hand, we argue that conservation of resources theory also explains the motivation of highly embedded employees towards TMX. In cognitive psychology, the salience of loss implies that, for individuals, losing resources is more psychologically destructive than gain (Halbesleben et al., 2014). Accordingly, highly embedded employees would bear higher level of risks of losing. Based on conservation of resources theory, the anxiety of losing resources, also the "difficulty" to leave as described in the concept of job embeddedness, would engage highly embedded employees in protecting the already established connections, and thus TMX, with peers in the organization.

Hypothesis 1. Job embeddedness is positively related to OCBI.

Hypothesis 2. Job embeddedness is positively related to TMX. 
We maintain that job embeddedness can facilitate employees' OCBI through enhanced TMX. Previous studies have shown empirical evidence to support the relationship between TMX and OCBI, and between job embeddedness and OCBI. For instance, in terms of the TMX and OCBI, Kamdar and Van Dyne (2007) noted that helping coworkers is naturally inherent in reciprocity, and individuals with highquality TMX tend to help coworkers to signal that they value their social exchange relationships. Farmer et al. (2015) took a different approach to assessing the effects of TMX on OCBI, and they separated the concept of TMX in between- and within-group levels, and they found out that the interactive effect of individual's relative TMX quality and group average TMX quality facilitate OCBI. In an effort to extend the cognition of job embeddedness as a predictor of retention, Lee et al. (2004) argued that, since job embeddedness represents the interdependencies within a workgroup, and helping behavior would be consistent with the "fit" to the social network, the highly embedded employees should display citizenship behavior, and their findings proved that job embeddedness was significantly predictive of OCBs. Also, in align with our theoretical arguments underlying the job embeddedness and TMX relationship, we propose that TMX mediates the job embeddedness and OCBI relationship, because not performing OCBI would break the reciprocity of social exchange. Further, in order to conserve resources and make social exchange going within the group, focal employees need to maintain high-quality relationships that contribute towards OCBI.

\section{Hypothesis 3. The positive relationship between job embeddedness and OCBI is mediated by TMX.}

\section{Outcome Orientation as the Moderator}

Another goal of our study is to investigate the role of workgroup climate for job embeddedness-TMX relationship, and embedded employees' behaviors towards peers. We emphasized on workgroup climate because it can constrain the conditional context to which individuals act upon their perceptions that further form attitudes and behaviors (Chen \& Kanfer, 2006). More specifically, we considered outcome orientation climate because this type of workgroup climate shapes the main stream value of a workgroup and underlines the outcome (O'Reilly et al., 1991). Outcome orientation describes workgroup context that advocates results rather than processes, and emphasizes high expectations for performance, and action, results and achievement (Liden, Anand, \& Vidyarthi, 2016; O'Reilly et al., 1991). Though theoretically matched with team orientation climate, which prioritizes collective effort, mutual trust, shared rewards, collective responsibilities, and group achievement (O'Reilly et al. (1991), outcome orientation with emphasis on individual achievement does not necessarily act as a counter "social exchange" climate. We argue that outcome orientated climate fosters awareness of resources for achievement and further enhances interactions between highly embedded individuals and peers.

Core to our argument is the relevance of outcome orientation to workgroup members' motivations toward resources accumulation and conservation. We expect that the climate of outcome orientation boosts peers' preference of resource accumulation and increases their tendency to exchange with highly embedded employees for resources. Considering that self-interests in outcome-oriented climate are commonly high, we expect that outcome orientation amplifies the motivation of resources conservation, and prevails the view that resources come from exchange.

Under outcome-oriented climate, workgroup members are expected to be more sensitive to intragroup differences in terms of status and resources, and peers are more prone to value their relationships with highly embedded employees. Under workgroup climate that advocates collective efforts and emphasizes on group outcomes, team members will provide support to each other regardless of intragroup differences, because they enact based on the collective norms rather than personal outcomes, and then they will not selectively choose to develop relationships with merit and resourceful individuals, such as highly embedded members (Chen, Huang, \& Tjosvold, 2008; Tjosvold, 1988). On the contrary, high outcome orientation rewards individual achievement and sensitizes workgroup members to performance above expectations. Hence, we foresee that resources would be highly valued in outcome-oriented climate. Drawing from conservation of resources theory, in workgroups with high outcome orientation, we expect that bonding with highly embedded employees would mean more to peers in terms of resources 
accumulation, and peers would be more motivated by such workgroup climate to provide more support for highly embedded employees as the means to develop the exchange relationship.

Correspondingly, we also expect that, in outcome-oriented climate, highly embedded employees who receive peers' support will follow the norm of reciprocity conceptualized in social exchange theory, and are obligated to perform helping behavior towards peers. In the outcome-oriented context, disregarding peers' support may create potential opponents in the workplace, and there will be less harm to highly embedded employees if their relationships with peers are based on rational exchange. Based on conservation of resources theory, when workgroup outcome orientation is high, we expect that the mediating effect of TMX will be more salient for the job embeddedness and OCBI relationship.

Hypothesis 4. The positive relationship between job embeddedness and TMX is moderated by the climate of outcome orientation, such that the relationship is stronger in groups with high outcome orientation compared to low outcome orientation.

Hypothesis 5. TMX mediates the relationship between job embeddedness and OCBI, such that this mediation effect is stronger in groups with high outcome orientation compared to low outcome orientation.

\section{METHODS}

\section{Data and Sample}

The study was conducted in a large private university in Asia. We used paper-and-pencil and online surveys to collect data. In total, we invited 450 employees for the study, and collected 348 responses (response rate, 77\%). Their 51 supervisors were contacted a month after we collected employee responses. Employees responded to the job embeddedness, and TMX measures, and their respective managers provided OCBI and outcome orientation for each employee and their workgroups.

We defined a workgroup in this study as having more than three employees. We excluded incomplete responses, and the final sample consisted of 297 employees (response rate, 66\%) and 49 supervisors (response rate, 96\%). In our final sample, 39.4\% were females. The average age was 38.9 years $(S D=$ $8.01)$, and average organizational tenure was 4.1 years $(S D=1.93)$ and dyadic tenure was $4.2(S D=$ 1.44). The average size of workgroup was about 8 employees.

\section{Measures}

All variables included in this study were measured with established scales used by previous empirical studies. Unless otherwise noted, items were measured on a 5-point Likert-type scale ranging from 1 (Strongly disagree) to 5 (Strongly agree).

$O C B I$

We used five-item measures of OCBI developed by Moorman and Blakely (1992). An example OCBI item is, "This employee goes out of his/her way to help co-workers with work-related problems." $(\alpha=$ $.94)$.

\section{Outcome Orientation}

We used O'Reilly et al.'s (1991) four-item measure of outcome orientation. An example item is, "achievement oriented," "action oriented," "high expectations," and "results oriented" $(\alpha=.94)$.

\section{Job Embeddedness}

We used Crossley et al.'s (2007) seven-item measure of job embeddedness. An example item is, "I simply could not leave the organization that I worked for" $(\alpha=.86)$. 
We used Liden et al.'s (2000) nine-item measure of TMX, which is based on Seers (1989). An example item is, "When I am in a bind, my co-workers will take on extra work to help ensure the completion of my important tasks." ( $\alpha=.76, \alpha=.88$ for Liden et al.'s original study).

\section{Control Variables}

For individual-level control variables, following Anand et al.'s (2010) study, which involves TMX and OCBI, we controlled employees' age, gender and organizational tenure and dyadic tenure with supervisor for the purpose to control for alternative explanations. We also controlled the number of employees' involved workgroups for its potential impact on the focal employees' TMX. For group-level control variable, following Vidyarthi et al.'s (2016) study that includes team climate variable, we controlled for group size.

\section{Analytical Strategy}

We adopted hierarchical linear modeling (Hofmann, Griffin, \& Gavin, 2000), because our theoretical model involves job embeddedness, TMX and OCBI as individual-level variables, and outcome orientation as group-level variables. Also, it was necessary to employ HLM because our data contains feedbacks from employees nested within groups, and from supervisors who rated OCBI and group-level outcome orientation. In addition, our proposed theoretical model consists of a 1-1-1 multilevel mediation model and a 1-1-1 multilevel moderated mediation model, and thus we used multilevel structural equation modeling (Preacher, Zyphur, \& Zhang, 2010; Song, 2018; Zhang, Zyphur, \& Preacher, 2009) with manifest variables and maximum likelihood estimation. We used Mplus 8.2 for testing mediation (Muthén \& Muthén, 2017).

\section{RESULTS}

Table 1 presents the means, standard deviations, and correlations among studied variables, and internal consistency reliabilities are in parentheses along the diagonal. Before hypothesis testing, we ran confirmatory factor analysis (CFAs) to assess the multiitem constructs. As illustrated in Table 2, the fitindices were acceptable for our hypothesized model: $\chi^{2}(269)=658.60, p<.05$; root mean square error of approximation $(\mathrm{RMSEA})=.07$; comparative fit index $(\mathrm{CFI})=.93$; root mean square residual $(\mathrm{SRMR})=$ .05. In order to assess the discriminate validity, we compared the hypothesized four-factor model with three alternate models. In the second model, items for job embeddedness and TMX loaded together on one factor. The fit-indices results were poorer in the second model: $\chi^{2}(270)=1276.73$, RMSEA $=.11$, $\mathrm{CFI}=.81, \mathrm{SRMR}=.09$. In the third model, items for OCBI and outcome orientation loaded together on one factor, and the fit-indices results were worse: $\chi^{2}(271)=2388.06, \mathrm{RMSEA}=.16, \mathrm{CFI}=.60, \mathrm{SRMR}=$ .14. In the fourth the one-factor model, fit-indices results were $\chi^{2}(272)=2682.78$, RMSEA $=.17$, CFI $=$ .55 , SRMR $=.19$. The fit-indices results for all alternative models demonstrated poorer fits to the data, and our hypothesized four-factor model best fit the data. 
TABLE 1

MEANS, STANDARD DEVIATIONS, AND INTERCORRELATIONS AMONG STUDY VARIABLES

\begin{tabular}{|c|c|c|c|c|c|c|c|c|c|c|}
\hline Variable & $M$ & $S D$ & 1 & 2 & 3 & 4 & 5 & 6 & 7 & 8 \\
\hline \multicolumn{11}{|l|}{ Individual-level variables } \\
\hline 1. Age & 38.92 & 8.01 & & & & & & & & \\
\hline 2. Gender & 1.39 & 0.49 & .07 & & & & & & & \\
\hline 3. OrganizationalTenure & 4.14 & 1.93 & $.23^{* *}$ & .06 & & & & & & \\
\hline 4. Dyadic tenure & 4.22 & 1.44 & $.39^{* *}$ & $.12^{*}$ & $.31^{* *}$ & & & & & \\
\hline 5. Workteam & 3.20 & 5.46 & .05 & .02 & $.22^{* *}$ & .08 & & & & \\
\hline 6. Job Embeddedness & 3.59 & 1.06 & .05 & $.14^{*}$ & $.27^{* *}$ & .04 & $.14^{*}$ & $(.86)$ & & \\
\hline 7. TMX & 4.06 & 0.61 & -.05 & .012 & -.05 & -.05 & .10 & $.36^{* *}$ & $(.76)$ & \\
\hline 8. OCBI & 3.28 & 0.97 & $.14^{*}$ & .02 & $.14^{*}$ & .11 & .02 & $.19^{* *}$ & $.19^{* *}$ & $(.94)$ \\
\hline \multicolumn{11}{|l|}{ Group-level variables } \\
\hline 1. Size & 7.69 & 2.50 & & & & & & & & \\
\hline 2. Outcome Orientation & 3.87 & 0.85 & .00 & $(.94)$ & & & & & & \\
\hline
\end{tabular}

TABLE 2

CFA RESULTS FOR HYPOTHESIZED AND ALTERNATE MODELS

\begin{tabular}{lrcccccc}
\hline \multicolumn{1}{c}{ Variable models } & \multicolumn{1}{c}{${ }^{2}$} & $d f$ & RMSEA & CFI & GFI & SRMR & $\Delta^{2}(\Delta \mathrm{df})$ \\
\hline 1. 4-factor & 658.60 & 269 & 0.07 & 0.93 & 0.86 & 0.05 & \\
2. 3-factor (combining job embeddedness and TMX) & 1276.73 & 270 & 0.11 & 0.81 & 0.68 & 0.09 & $618.13(1)^{* *}$ \\
3. 2-factor (combining OCBI and outcome orientation) & 2388.06 & 271 & 0.16 & 0.60 & 0.57 & 0.14 & $1111.33(1)^{* *}$ \\
4. 1-factor & 2682.78 & 272 & 0.17 & 0.55 & 0.52 & 0.19 & $294.72(1)^{* *}$ \\
\hline
\end{tabular}

$* p<.05 . * * p<.01$. RMSEA $=$ root-mean-square error of approximation; $\mathrm{CFI}=$ comparative fit index; GFI $=$ goodness of fit index; SRMR = root mean square residual.

Before hypotheses testing, we developed null models to estimate the variability in TMX and OCBI attributable to group membership. The ratio of the between-group variance in TMX to the total variance produced an ICC(1) of .101, indicating that $10.1 \%$ of total variance in TMX was due to group membership. For OCBI, the ratio of the between-group variance to the total variance yielded an ICC(1) of .256 , suggesting that $25.6 \%$ of total variance in OCBI was attributable to the nesting effect. According to Bliese (2000), both ICC(1) values were over the recommended criteria of $10 \%$, and the use of HLM was justified for controlling for group membership effects.

We first ran Model 1 with control variables: age, sex, organizational tenure, dyadic tenure, work team at Level 1 and group size at Level 2. In Model 2 we added job embeddedness as a Level 1 predictor. Hypothesis 2 suggested that job embeddedness is positively associated with TMX. As indicated in Model 2 of Table 3, the results showed a positive and significant coefficient for job embeddedness in predicting $\operatorname{TMX}(\gamma=.28, p<.01)$. The Akaike information criterion (AIC) indicated a significant improvement in fit compared to the null model $(\triangle \mathrm{AIC}=55.02) . R^{2}$ showed that job embeddedness explained $22 \%$ of variance in TMX. Hypothesis 1 stated that job embeddedness is positively related to OCBI. After controlling age, sex, organizational tenure, dyadic tenure, work team at Level 1 and group size at Level 2 in Model 6, we observed a significant positive association between job embeddedness and OCBI $(\gamma=.21$, $p<.01$ ), which supported hypothesis 1 . 


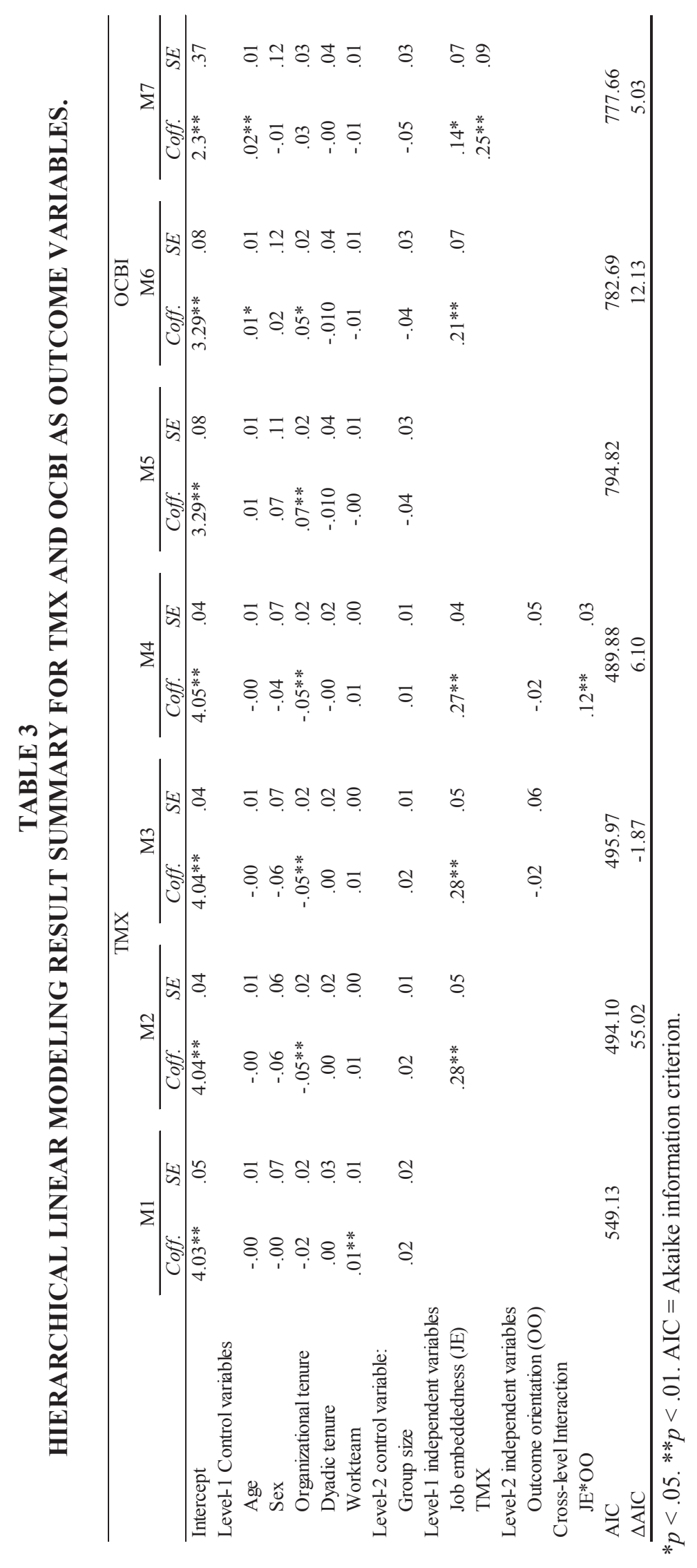

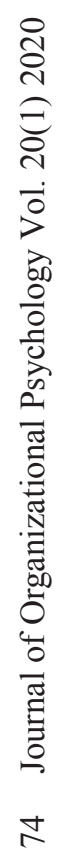


Hypothesis 4 stated that outcome orientation moderates the relationship between job embeddedness and TMX, such that job embeddedness is more positively associated with TMX when outcome orientation is high rather than low. To test hypothesis 4 regarding the cross-level moderating effect of group-level outcome orientation, we added outcome orientation as a control in Model 3 to create Model 4. The result reported in Model 4 of Table 3 showed that the effect of job embeddedness was dependent on outcome orientation: the interaction term for job embeddedness and outcome orientation was positive and significant $(\gamma=.12, p<.01)$, and thus supported hypothesis 4 . A significant improvement in model fit was shown by reduction in AIC $(\triangle \mathrm{AIC}=6.10)$. Changes in $R^{2}$ suggested that the interaction term accounted for $.51 \%$ Level 1 variance in TMX. Following the support for the moderating effect of team orientation, we plotted the relationship between job embeddedness and TMX for low and high levels of group level outcome orientation in Figure 2.

\section{FIGURE 2 \\ MODERATION EFFECT OF OUTCOME ORIENTATION ON JOB EMBEDDEDNESS-TMX RELATIONSHIP}



For testing hypothesis 3 and hypothesis 5 we applied the 1-1-1 multilevel mediation and 1-1-1 multilevel moderated mediation test using MSEM in Mplus. Hypothesis 3 stated that TMX mediates the relationship between job embeddedness and OCBI. In Table 3, there was a significant positive association between TMX and OCBI $(\gamma=.25, p<.01)$, and after introducing TMX at Level 1, the coefficient for job embeddedness in predicting OCBI became less significant $(\gamma=.14, p<.05)$, which indicated promising mediation (compare Model 6 and 7).

To avoid the potential biases that innate in the estimations of indirect effects of clustered data, which are omitted in the conventional 'conflated' multilevel modeling approaches, we followed suggestions provided by Zhang et al. (2009) and Preacher et al. (2010), and conducted deconflated multilevel modeling path analyses in Mplus 8. This approach has been adopted in other studies that focused on the assessment of multilevel indirect effects (e.g., Huang, Chiaburu, Zhang, Li, \& Grandey, 2015; Uy, Lin, \& Ilies, 2017). For Level 1 variables job embeddedness and TMX, we used group mean-centering. Hypothesis 3 stated that TMX mediates the relationship between job embeddedness and OCBI. The results in Table 4 showed that the direct effect of job embeddedness on OCBI was not statistically significant at the within-level (coeff $=.13, S E=.09, n s)$, nor at the between-level (coeff $=.09, S E=.18$, $n s)$, and the indirect effect through TMX was statistically significant at the within-level (coeff $=.07, S E=$ $.04, p<.05)$, but not at the between-level $($ coeff $=.12, S E=.09, n s)$, supporting hypothesis 3 (see Table $4)$. 
TABLE 4

1-1-1 MLM MEDIATION

\begin{tabular}{lccc}
\hline & Coeff. & SE & $p$ \\
\hline Direct effect, between-level & .09 & .18 & .62 \\
Direct effect, within-level & .13 & .09 & .16 \\
Indirect effect, between-level & .12 & .09 & .18 \\
Direct effect, within-level & $.07 *$ & .04 & .05 \\
\hline$* p<.05$. & &
\end{tabular}

Hypothesis 5 stated that the indirect effect of job embeddedness on OCBI through TMX is more positive when outcome orientation is high. Following the same analytical strategy used to assess 1-1-1 mediation, we used the deconflated multilevel modeling approach. Also, we computed the conditional values of the Level-2 moderator outcome orientation one standard deviation above and below the mean, and then added these two conditional values and the mean value into the equation of the 1-1-1 multilevel mediation model. As shown in Table 5, we found that the conditional indirect effect was positive and significant at the within-level when outcome orientation was high $($ coeff $=.10, S E=.05, p<.05)$, but positive and insignificant when outcome orientation was low (coeff $=.05, S E=.03, n s)$, and the direct effects at the within-level were insignificant when outcome orientation was low (coeff $=.13, S E=.19, n s)$ and high $($ coeff $=.13, S E=.09, n s)$. These results supported hypothesis 5 (see Table 5).

TABLE 5

\section{1-1-1 MLM MODERATED MEDIATION}

\begin{tabular}{|c|c|c|c|c|c|c|}
\hline & \multicolumn{3}{|c|}{ Direct effect, between-level } & \multicolumn{3}{|c|}{ Direct effect, within-level } \\
\hline & Coeff. & $S E$ & $p$ & Coeff. & $S E$ & $p$ \\
\hline Outcome Orientationn (-1 SD) & \multirow{2}{*}{.06} & \multirow{2}{*}{.20} & \multirow{2}{*}{.76} & .13 & .09 & .13 \\
\hline \multirow[t]{3}{*}{ Outcome Orientation $(+1 S D)$} & & & & .13 & .09 & .13 \\
\hline & \multicolumn{3}{|c|}{ Indirect effect, between-level } & \multicolumn{3}{|c|}{ Indirect effect, between-leve } \\
\hline & Coeff. & $S E$ & $p$ & Coeff. & $S E$ & $p$ \\
\hline Outcome Orientationn (-1 SD) & \multirow{2}{*}{.12} & \multirow{2}{*}{.09} & \multirow{2}{*}{.17} & .05 & .03 & .11 \\
\hline Outcome Orientation $(+1 S D)$ & & & & $.10^{*}$ & .05 & .04 \\
\hline
\end{tabular}

${ }^{*} p<.05$.

\section{DISCUSSION}

Current understanding of job embeddedness and its effectiveness are constrained by its original concept. Previous research on the job embeddedness-OCBs focused largely on the predicting role of job embeddedness, but did not explore further the potential mechanisms underlying such relationship, as well as situational factors. The aim of this study, therefore, was to use two theoretical lenses, namely, social exchange theory and conservation of resources theory, to better understand the social influence of highly embedded individuals on their relationships with rest of the workgroup members in an outcome-oriented workgroup climate.

We emphasized on OCBI, a specific form of OCBs that can immediately benefit workgroup members, and provided a novel explanation on the formation of TMX in which highly embedded individuals are centered, and the mediating effect of TMX that transmits highly embedded individuals' decision to perform OCBI. We also tested whether the job embeddedness-TMX-OCBI mediation relationship will be persistent in an outcome-oriented climate - an important workgroup climate 
advocating results and achievement, which provided an ideal situational context to test our assumption that eager for resources is a key motive for peers to cooperate with highly embedded individuals.

Overall, our results largely supported hypothesized relationships: TMX transmitted the positive effect of job embeddedness on OCBI, and the relationship between job embeddedness and TMX, and the indirect effects through TMX, were stronger for workgroups advocating outcome orientation. These findings are aligned with job embeddedness theory, because we expect highly embedded individuals to be identified by their peers as the foci of resources. In order to become the beneficiary of highly embedded employees' resources, less embedded employees are motivated to build up high quality relationship with those focal individuals. On the other hand, highly embedded employees would not voluntarily and nonselectively demonstrate helping behaviors, and they would only do so to peers who they believe would also help them and develop good quality relationships with them. In an outcome-oriented workgroup climate, highly embedded employees become more salient to rest of the peers because of the high demand on results achievement. When goals become harder and require more resources to complete, less embedded peers will seek help from highly embedded employees; however, since highly embedded individuals are also motivated to conserve or develop resources for goal achievement, less embedded peers would need to join those focal individuals, and exchange their "friendliness" for highly embedded employees' help.

Results of our study have several important theoretical implications. First, this study is the first empirical study to integrate job embeddedness and TMX: we theorized and utilized TMX as the mediator to job embeddedness and OCBI relationship, and developed and tested a multi-level mediation model. We have shown that job embeddedness is positively related to TMX, and TMX fully mediates the job embeddedness-OCBI relationship. The overall findings have substantial implications for advancing the current understanding of the formation of TMX in a workgroup setting, and the mechanism underlying job embeddedness and OCBI. We contribute to the theoretical enrichment of both job embeddedness and OCBs by specifying the effectiveness of job embeddedness on OCBI with TMX as the hinge to this relationship.

Second, our findings shed light on the moderating role of outcome orientation in the job embeddedness and TMX relationship. Outcome orientation in theory is more of an "ends justify the means" team climate. Although it prevails high expectations for performance and individual achievement, and deprioritizes collective effort, it is not explicitly a negative workgroup variable (Cole et al., 2002; O'Reilly et al., 1991), and the ambiguity of its effects may explain its absence in empirical studies. Founding on conservation of resources theory and social exchange theory, we boldly predicted that, in outcome-oriented workgroup climate, the positive relationship between job embeddedness and TMX will be strengthened, and the mediating effect of TMX on job embeddedness and OCBI will be amplified. These hypotheses were supported by our results. Thus, our study makes contributions to the existing understanding of outcome ordination by offering important empirical evidence.

Finally, past research has shown job embeddedness as an antecedent to OCBs (Lee et al., 2004). We looked further into this relationship by hypothesizing that the positive relationship between job embeddedness and OCBI will be weakened by outcome orientation. Our results first replicated the job embeddedness and OCBI relationship. After we introduced the interaction term of outcome orientation and job embeddedness, the job embeddedness and OCBI relationship became weaker but not significantly changed by interaction effect. This finding echoed previous argument that job embeddedness implies workgroup interdependencies, and highly embedded employees will be motivated to perform OCBs because of their connections in the organization (Lee et al., 2004). Our findings may indicate that the job embeddedness and OCBI relationship will be constant regardless of contextual exchanges. To verify this assumption, we controlled TMX, and our results showed a negative and significant coefficient of the outcome orientation and job embeddedness interaction term on OCBI. This finding reconfirms the importance of TMX in job embeddedness and OCBI relationship, which implies that relationships with peers create the crucial incentive for highly embedded employees to perform OCBI. 


\section{Limitations and Future Research Directions}

One of the limitations of this study is that we only included outcome orientation as the group-level moderator. Other group level factors, for instance, task independence or group cohesiveness (Liden et al., 2016; Vidyarthi et al., 2016), could also provide conditional context that alters attitudes and behaviors of both highly embedded employees and their peers. Therefore, future researchers should investigate the impacts of different workgroup climates on job embeddedness, TMX and OCBI relationships.

Second, despite that we theoretically claimed the causal relationships of job embeddedness, TMX and OCBI, the cross-sectional design of our study is not sufficient to support such claim. As Holtom and Sekiguchi (2018) suggested, job embeddedness and OCBs may be mutually reinforcing, and OCBs could develop network ties (links), liking and trust (fit) and appropriability of network ties (sacrifice) that determine job embeddedness. Thus, future researchers should adopt longitudinal design to examine job embeddedness, TMX and OCBs to establish the causalities.

Last but not least, the data used in this study were collected from a large private university in Asia, and it is legitimate to question the generalizability of our findings. Future researchers should attempt to replicate our models in different industries and countries. In addition, in the current study we adopted the global measure of job embeddedness from Crossley et al. (2007). Future studies should also test the job embeddedness-TMX-OCBI relationship based on Mitchell et al.'s (2001) composite measure, even though both measures have been found to produce a generally similar pattern of results on withdrawal behaviors, it is important to further validate whether the composite measure of job embeddedness can generate the same results.

\section{Practical Implications}

Our study has important practical implications for the management of interpersonal exchange processes and cooperation in workgroups. Even though team orientation promotes collective efforts (Tsui, Egan, \& O'Reilly, 1992; Vidyarthi et al., 2016), and outcome orientation focuses on results rather than processes, our findings suggest that outcome orientated climate is not necessarily opposite to team oriented climate, and it may be more efficient in terms of utilizing personal resources, because outcome orientated climate facilitated cooperation around highly embedded employees with valued resources, but not among less embedded employees who have less valued resources. Therefore, we suggest that organizations can develop small and medium size project teams around several highly embedded employees to foster effective cooperation for specific goal achievement.

\section{CONCLUSION}

The present study contributes to the research on job embeddedness and OCBs relationship by developing and testing a multilevel moderated mediation model that includes TMX, which mediates the job embeddedness-OCBI relationship at individual level, and outcome orientation, which moderates the job embeddedness-TMX relationship, and the mediating role of TMX, at the group level. Results help to explain highly embedded employees are likely to elicit helping behaviors towards peers because they are able to maintain high quality relationships with workgroup members. Further they see maintaining these relationships more valuable in high outcome-oriented workgroup climates. We hope that our findings stimulate future researchers to pay closer attention to the job embeddedness and OCBs relationship, and explore other mediating variables, and design and conduct research with different approaches and in different contexts to uncover other explanations. 


\section{REFERENCES}

Anand, S., Vidyarthi, P. R., Liden, R. C., \& Rousseau, D. M. (2010). Good citizens in poor-quality relationships: idiosyncratic deals as a substitute for relationship quality. Academy of Management Journal, 53(5), 970-988.

Baird, K., Jia Hu, K., \& Reeve, R. (2011). The relationships between organizational culture, total quality management practices and operational performance. International Journal of Operations \& Production Management, 31(7), 789-814.

Banks, G. C., Batchelor, J. H., Seers, A., O'Boyle, E. H., Pollack, J. M., \& Gower, K. (2014). What does team-member exchange bring to the party? A meta-analytic review of team and leader social exchange. Journal of Organizational Behavior, 35(2), 273-295.

Blau, P. (1964). 1964 Exchange and power in social life. New York: Wiley.

Bliese, P. D. (2000). Within-group agreement, non-independence, and reliability: Implications for data aggregation and analysis. San Francisco, CA, US: Jossey-Bass.

Campbell, E. M., Liao, H., Chuang, A., Zhou, J., \& Dong, Y. (2017). Hot shots and cool reception? An expanded view of social consequences for high performers. Journal of Applied Psychology, 102(5), 845-866.

Chen, G., \& Kanfer, R. (2006). Toward a systems theory of motivated behavior in work teams. Research in Organizational Behavior, 27, 223-267.

Chen, Y-F., Huang, X., \& Tjosvold, D. (2008). Similarity in gender and self-esteem for supportive peer relationships: the mediating role of cooperative goals. Journal of Applied Social Psychology, 38(5), 1147-1178.

Chiaburu, D. S., \& Harrison, D. A. (2008). Do peers make the place? Conceptual synthesis and metaanalysis of coworker effects on perceptions, attitudes, OCBs, and performance. Journal of Applied Psychology, 93(5), 1082-1103.

Cole, M. S., Schaninger, W. S., Jr., \& Harris, S. (2002). The workplace social exchange network: A multilevel, conceptual examination. Group \& Organization Management, 27(1), 142-167.

Cropanzano, R., \& Mitchell, M. S. (2005). Social exchange theory: an interdisciplinary review. Journal of Management, 31(6), 874-900.

Crossley, C. D., Bennett, R. J., Jex, S. M., \& Burnfield, J. L. (2007). Development of a global measure of job embeddedness and integration into a traditional model of voluntary turnover. Journal of Applied Psychology, 92(4), 1031-1042.

Eisenberger, R., Huntington, R., Hutchison, S., \& Sowa, D. (1986). Perceived organizational support. Journal of Applied Psychology, 71(3), 500-507.

Eisenberger, R., Stinglhamber, F., Vandenberghe, C., Sucharski, I. L., \& Rhoades, L. (2002). Perceived supervisor support: Contributions to perceived organizational support and employee retention. Journal of Applied Psychology, 87(3), 565-573.

Farmer, S. M., Van Dyne, L., \& Kamdar, D. (2015). The contextualized self: How team-member exchange leads to coworker identification and helping OCB. Journal of Applied Psychology, $100(2), 583-595$.

Gouldner, A. W. (1960). The norm of reciprocity: a preliminary statement. American Sociological Review, 25(2), 161-178.

Grant, A. M., \& Parker, S. K. (2009). Redesigning Work Design Theories: The Rise of Relational and Proactive Perspectives. The Academy of Management Annals, 3(1), 317-375.

Griffin, M. A., Neal, A., \& Parker, S. K. (2007). A New Model of Work Role Performance: Positive Behavior in Uncertain and Interdependent Contexts. Academy of Management Journal, 50(2), 327-347.

Halbesleben, J. R. B., \& Bowler, W. M. (2007). Emotional exhaustion and job performance: The mediating role of motivation. Journal of Applied Psychology, 92(1), 93-106. 
Halbesleben, J. R. B., Neveu, J-P., Paustian-Underdahl, S. C., \& Westman, M. (2014). Getting to the "COR": understanding the role of resources in conservation of resources theory. Journal of Management, 40(5), 1334-1364.

Hobfoll, S. E. (1989). Conservation of resources: A new attempt at conceptualizing stress. American Psychologist, 44(3), 513-524.

Hobfoll, S. E. (2001). The influence of culture, community, and the nested-self in the stress process: advancing conservation of resources theory. Applied Psychology, 50(3), 337-421.

Hofmann, D. A., Griffin, M. A., \& Gavin, M. B. (2000). The application of hierarchical linear modeling to organizational research. In K. J. Klein \& S. W. J. Kozlowski (Eds.), Multilevel theory, research, and methods in organizations: Foundations, extensions, and new directions. (pp. 467511). San Francisco, CA, US: Jossey-Bass.

Holtom, B. C., \& Sekiguchi, T. (2018). Exploring the relationship between job embeddedness and organizational citizenship behavior. In P. M. Podsakoff, S. B. MacKenzie, \& N. P. Podsakoff (Eds.), The Oxford Handbook of Organizational Citizenship Behavior (pp. 371): Oxford University Press.

Homans, G. C. (1958). Social behavior as exchange. American Journal of Sociology, 63(6), 597-606.

Huang, J. L., Chiaburu, D. S., Zhang, X-A., Li, N., \& Grandey, A. A. (2015). Rising to the challenge: Deep acting is more beneficial when tasks are appraised as challenging. Journal of Applied Psychology, 100(5), 1398-1408.

Kamdar, D., \& Van Dyne, L. (2007). The joint effects of personality and workplace social exchange relationships in predicting task performance and citizenship performance. Journal of Applied Psychology, 92(5), 1286-1298.

Lee, T. W., Mitchell, T. R., Sablynski, C. J., Burton, J. P., \& Holtom, B. C. (2004). The effects of job embeddedness on organizational citizenship, job performance, volitional absences, and voluntary turnover. Academy of Management Journal, 47(5), 711-722.

Liden, R. C., Anand, S., \& Vidyarthi, P. (2016). Dyadic relationships. Social Science Electronic Publishing, 3(1).

Liden, R. C., Wayne, S. J., \& Sparrowe, R. T. (2000). An examination of the mediating role of psychological empowerment on the relations between the job, interpersonal relationships, and work outcomes. Journal of Applied Psychology, 85(3), 407-416.

Lincoln, J. R., \& Miller, J. (1979). Work and Friendship Ties in Organizations: A Comparative Analysis of Relational Networks. Administrative Science Quarterly, 24(2), 181-199.

Love, M. S., \& Forret, M. (2008). Exchange relationships at work: An examination of the relationship between team-member exchange and supervisor reports of organizational citizenship behavior. Journal of Leadership \& Organizational Studies, 14(4), 342-352.

Mitchell, T. R., Holtom, B. C., Lee, T. W., Sablynski, C. J., \& Erez, M. (2001). Why people stay: using job embeddedness to predict voluntary turnover. Academy of Management Journal, 44(6), 1102 1121.

Moorman, R., \& Blakely, G. (1992). A preliminary report on a new measure of organizational citizenship behavior. Paper presented at the Proceedings of the annual meeting of the southern management association.

Muthén, L. K., \& Muthén, B. O. (2017). Mplus: Statistical analysis with latent variables: User's guide. Muthén \& Muthén Los Angeles.

O'Reilly, C. A., Chatman, J., \& Caldwell, D. F. (1991). People and organizational culture: a profile comparison approach to assessing person-organization fit. Academy of Management Journal, 34(3), 487-516.

Podsakoff, P. M., MacKenzie, S. B., Paine, J. B., \& Bachrach, D. G. (2000). Organizational citizenship behaviors: A critical review of the theoretical and empirical literature and suggestions for future research. Journal of Management, 26(3), 513-563.

Preacher, K. J., Zyphur, M. J., \& Zhang, Z. (2010). A general multilevel SEM framework for assessing multilevel mediation. Psychological Methods, 15(3), 209-233.

80 Journal of Organizational Psychology Vol. 20(1) 2020 
Robbins, J. M., Ford, M. T., \& Tetrick, L. E. (2012). Perceived unfairness and employee health: A metaanalytic integration. Journal of Applied Psychology, 97(2), 235-272.

Scott, S. G., \& Bruce, R. A. (1994). Determinants of innovative behavior: a path model of individual innovation in the workplace. The Academy of Management Journal, 37(3), 580-607.

Seers, A. (1989). Team-member exchange quality: A new construct for role-making research. Organizational Behavior and Human Decision Processes, 43(1), 118-135.

Song, H. (2018). A primer on multilevel mediation models for egocentric social network data. Communication Methods and Measures, 12(1), 1-24.

Tjosvold, D. (1988). Cooperative and competitive dynamics within and between organizational units. Human Relations, 41(6), 425-436.

Tse, H. H. M., Dasborough, M. T., \& Ashkanasy, N. M. (2008). A multi-level analysis of team climate and interpersonal exchange relationships at work. The Leadership Quarterly, 19(2), 195-211.

Tsui, A. S., Egan, T. D., \& O'Reilly, C. A. (1992). Being different: relational demography and organizational attachment. Administrative Science Quarterly, 37(4), 549-579.

Uy, M. A., Lin, K. J., \& Ilies, R. (2017). Is it better to give or receive? The role of help in buffering the depleting effects of surface acting. Academy of Management Journal, 60(4), 1442-1461.

Vidyarthi, P. R., Singh, S., Erdogan, B., Chaudhry, A., Posthuma, R., \& Anand, S. (2016). Individual deals within teams: Investigating the role of relative i-deals for employee performance. Journal of Applied Psychology.

Williams, L. J., \& Anderson, S. E. (1991). Job satisfaction and organizational commitment as predictors of organizational citizenship and in-role behaviors. Journal of Management, 17(3), 601-617.

Yao, X., Lee, T. W., Mitchell, T. R., Burton, J. P., \& Sablynski, C. S. (2004). Job embeddedness: Current research and future directions. In R. Griffeth \& P. Horn (Eds.), Understanding employee retention turnover (pp. 153-187). Creenwich CT: Information Age.

Zhang, Y., \& Chen, J. (2010, August 24-26). Motivational Mechanism Research of On-the-Job Embeddedness Effects on Intentions to Stay. Paper presented at the 2010 International Conference on Management and Service Science.

Zhang, Z., Zyphur, M. J., \& Preacher, K. J. (2009). Testing multilevel mediation using hierarchical linear models: problems and solutions. Organizational Research Methods, 12(4), 695-719. 